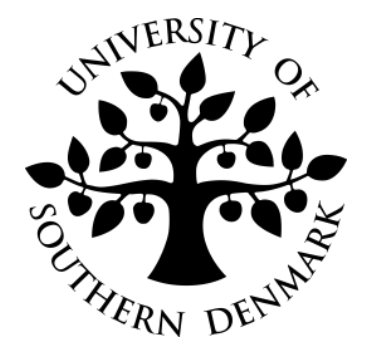

\title{
A Note on Testing the LATE Assumptions
}

by

\section{Lukas Laffers and Giovanni Mellace}

Discussion Papers on Business and Economics

No. 4/2015

FURTHER INFORMATION Department of Business and Economics Faculty of Business and Social Sciences University of Southern Denmark Campusvej 55, DK-5230 Odense M Denmark 


\title{
A Note on Testing the LATE Assumptions
}

\author{
Lukas Laffers* Giovanni Mellace ${ }^{\dagger}$
}

March 2, 2015

\begin{abstract}
In this paper we show that the testable implications derived in Huber and Mellace (2013) are the best possible to detect invalid instruments, in the presence of heterogeneous treatment effects and endogeneity. We also provide a formal proof of the fact that those testable implication are only necessary but not sufficient conditions for instrument validity.
\end{abstract}

Keywords: Testing IV validity, Local average treatment effect, Moment inequalities, bounds.

JEL classification: C12, C21, C26.

\section{Introduction}

In heterogeneous treatment effect models with a binary treatment, a binary instrument is valid (it allows identifying the Local Average Treatment Effect on the compliers) if (i) the potential outcomes are mean independent of the instrument, (ii) the types are independent on the instruments, and (iii) the potential treatment states are weakly monotonic function of the instrument. Huber and Mellace (2013) derive testable implication of those three assumptions. The main intuition is that under (i) to (iii), the mean potential outcomes of the always takers under treatment and the never takers under non-treatment can be both point identified and bounded. As the point identified mean potential outcomes must lie between the respective bounds this result provides four testable implications. However, Huber and Mellace (2013) do not provide any formal proof that those testable implications are the best possible to detect invalid instrument.

In this paper we show that those testable implications are indeed optimal in the sense of Preposition 1.1 in Kitagawa (2014). In particular, we show that for any observed joint distribution of the outcome, the treatment, and the instrument which satisfy the testable implications,

\footnotetext{
${ }^{*}$ Matej Bel University, Dept. of Mathematics, E-mail: lukas.laffers@gmail.com, Web: http://www.lukaslaffers.com

${ }^{\dagger}$ University of Southern Denmark, Dept. of Business and Economics, E-mail: giome@sam.sdu.dk, Web: http://sites.google.com/site/giovannimellace/
} 
there exist a DGP which satisfies assumptions (i) to (iii) and is compatible with such joint distribution. Moreover, we formally prove that it is only possible to refute but not to verify (i) to (iii), regardless of the sample size.

\section{Notation}

In this section we follow closely the notation in Huber and Mellace (2013). We denote by $Y$ the observed outcome, by $D$ the binary treatment and by $Z$ the binary instrument. We define the potential outcomes as $Y^{d}$ and the potential treatment states as $D^{z}$, respectively. ${ }^{1}$ As shown in the seminal papers of Imbens and Angrist (1994) and Angrist et al. (1996), the population can then be categorized into four types (denoted by $T$ ), as reported in Table 1 .

Table 1: Types

\begin{tabular}{c|cc|c}
\hline \hline Type $T$ & $D^{1}$ & $D^{0}$ & Notion \\
\hline$a$ & 1 & 1 & Always takers \\
$c$ & 1 & 0 & Compliers \\
$d f$ & 0 & 1 & Defiers \\
$n$ & 0 & 0 & Never takers \\
\hline
\end{tabular}

As either $D^{1}$ or $D^{0}$ is observed, but never both, without further assumptions, neither the share of the different types nor their mean potential outcomes are identified. Without loss of generality assume that $\operatorname{Pr}(D=1 \mid Z=1)>\operatorname{Pr}(D=1 \mid Z=0)$, the Local Average Treatment Effect (LATE) on the compliers is identified under the following three assumptions: ${ }^{2}$

Assumption 1: (unconfounded type)

$\operatorname{Pr}(T=t \mid Z=1)=\operatorname{Pr}(T=t \mid Z=0)$ for $t \in\{a, c, d f, n\}$.

Assumption 2: (mean exclusion restriction)

$E\left(Y^{d} \mid T=t, Z=1\right)=E\left(Y^{d} \mid T=t, Z=0\right)$ for $d \in\{0,1\}$ and $t \in\{a, c, d f, n\}$.

Assumption 3: (monotonicity)

$\operatorname{Pr}\left(D^{1} \geq D^{0}\right)=1$

Under Assumptions 1, and 3, the probability of being a defier is equal to zero and the probabilities of belonging to any other type is identified and do not depend on the instrument. Thus, let $\pi_{t} \equiv \operatorname{Pr}(T=t), t \in\{a, c, d f, n\}$, represent the probability of belonging to type $T$ in the

\footnotetext{
${ }^{1}$ By defining the potential outcomes, we implicitly assume that the usual Stable Unit Treatment Value assumption (SUTVA) holds.

${ }^{2}$ If $\operatorname{Pr}(D=1 \mid Z=1)<\operatorname{Pr}(D=1 \mid Z=0)$ one can run the test on $\tilde{Z}=1-Z$.
} 
population and denote by $P_{d \mid z} \equiv \operatorname{Pr}(D=d \mid Z=z)$ the conditional probability of being treated given the instrument, the implications of Assumptions 1 and 3 are summarized in Table 2.

Table 2: Observed probabilities and type proportions

\begin{tabular}{c|c}
\hline \hline Cond. treatment prob. & type proportions \\
\hline$P_{1 \mid 1} \equiv \operatorname{Pr}(D=1 \mid Z=1)$ & $\pi_{a}+\pi_{c}$ \\
$P_{0 \mid 1} \equiv \operatorname{Pr}(D=0 \mid Z=1)$ & $\pi_{n}$ \\
$P_{1 \mid 0} \equiv \operatorname{Pr}(D=1 \mid Z=0)$ & $\pi_{a}$ \\
$P_{0 \mid 0} \equiv \operatorname{Pr}(D=0 \mid Z=0)$ & $\pi_{c}+\pi_{n}$ \\
\hline
\end{tabular}

Similarly, under Assumptions 1, 2, and 3 and using the results in Table 2, we can relate the four observable conditional means, $E(Y \mid D=d, Z=z)$, to the types mean potential outcomes:

$$
\begin{aligned}
& E(Y \mid D=1, Z=1)=\frac{P_{1 \mid 0}}{P_{1 \mid 1}} \cdot E\left(Y^{1} \mid T=a\right)+\frac{P_{1 \mid 1}-P_{1 \mid 0}}{P_{1 \mid 1}} \cdot E\left(Y^{1} \mid T=c\right), \\
& E(Y \mid D=1, Z=0)=E\left(Y^{1} \mid T=a\right), \\
& E(Y \mid D=0, Z=1)=E\left(Y^{0} \mid T=n\right), \\
& E(Y \mid D=0, Z=0)=\frac{P_{1 \mid 1}-P_{1 \mid 0}}{P_{0 \mid 0}} \cdot E\left(Y^{0} \mid T=c\right)+\frac{P_{0 \mid 1}}{P_{0 \mid 0}} \cdot E\left(Y^{0} \mid T=n\right),
\end{aligned}
$$

Let $q_{r} \equiv \frac{P_{r \mid 1-r}}{P_{r \mid r}}$ and $y_{q_{r}}=F_{Y \mid D=r, Z=r}^{-1}\left(q_{r}\right)$ with $F$ being the cdf of $Y$ given $D=r, Z=r$ and $r=0,1$, using the results in Horowitz and Manski (1995), Huber and Mellace (2013) have shown that

$$
E\left(Y \mid D=r, Z=r, Y \leq y_{q_{r}}\right) \leq E(Y \mid D=r, Z=1-r) \leq E\left(Y \mid D=r, Z=r, Y \geq y_{1-q_{r}}\right)
$$

This provides four testable implications which are used by Huber and Mellace (2013) to jointly test the validity of Assumptions 1, 2, and 3.

\section{Formal proof of optimality and non-verifiability}

In this section we show that the inequalities in (2) are the best possible to screen invalid instrument, and that Assumptions 1, 2 and 3 are refutable but non-verifiable in the sense of Preposition 1.1 in Kitagawa (2014). 
Theorem 1. The following statements hold.

(i) For any probability distribution of the observed variables $(Y, D, Z)$ that satisfies the set of inequalities in (2), there exists a probability distribution of $\left(Y^{1}, Y^{0}, T, Z\right)$ that satisfies the Assumptions 1,2 and 3, and induces the observed probability distribution of $(Y, D, Z)$.

(ii) For any probability distribution of the observed variables $(Y, D, Z)$ satisfying $(2)$, there exists a probability distribution of $\left(Y^{1}, Y^{0}, T, Z\right)$ such that $Z$ is not a valid instrument.

Part (i) states that the inequalities in (2) are the best possible to detect violations of Assumptions (1) to (3), (ii) shows that the fact that (2) holds does not guarantee that the instrument is valid. The proof of Theorem 1 is presented below and is based on the results in Huber et al. (2014).

\section{Proof of theorem 1. Part (i)}

We decompose the probability distribution of $\left(Y^{1}, Y^{0}, T, Z\right)$ into the distribution of $\left(Y^{1}, Y^{0}\right)$ given $T$ and $Z$ and the probability distribution of $T$ given $Z$. The latter is uniquely determined under Assumptions 3 and does not depend on $Z$ (See Table 2). We note that the marginal distribution of $Z$ does not play any role in the model assumptions. Let $h_{t}^{z}\left(y^{1}, y^{0}\right)=f\left(y^{1}, y^{0} \mid T=\right.$ $t, Z=z)$ be the conditional density of $\left(Y^{1}, Y^{0}\right)$ evaluated at $\left(y^{1}, y^{0}\right)$ given $T=t$ and $Z=z$. For the sake of brevity, we refer to $h_{t}^{z}\left(y^{1}, y^{0}\right)$ by $h_{t}^{z}$. Let $g(y(d))$ denotes an arbitrary probability density function of $Y^{d}$. Consider the following specification for $h_{t}^{z}$ :

$$
\begin{aligned}
h_{a}^{1} & =g\left(y^{0}\right) \cdot\left(\alpha_{a}^{1} f_{Y}\left(y^{1} \mid D=1, Z=1, Y \leq y_{q_{1}}\right)+\left(1-\alpha_{a}^{1}\right) f_{Y}\left(y^{1} \mid D=1, Z=1, Y \geq y_{1-q_{1}}\right)\right), \\
h_{a}^{0} & =g\left(y^{0}\right) \cdot f_{Y}\left(y^{1} \mid D=1, Z=0\right), \\
h_{n}^{1} & =g\left(y^{1}\right) \cdot f_{Y}\left(y^{0} \mid D=0, Z=1\right), \\
h_{n}^{0} & =g\left(y^{1}\right) \cdot\left(\alpha_{n}^{0} f_{Y}\left(y^{0} \mid D=0, Z=0, Y \leq y_{q_{0}}\right)+\left(1-\alpha_{n}^{0}\right) f_{Y}\left(y^{0} \mid D=0, Z=0, Y \geq y_{1-q_{0}}\right)\right), \\
h_{c}^{1} & =h_{c}^{0}=\left(P_{1 \mid 1}-P_{1 \mid 0}\right)^{-2} \cdot\left(P_{1 \mid 1} \cdot f_{Y}\left(y^{1} \mid D=1, Z=1\right)-P_{1 \mid 0} \cdot \int h_{a}^{1} d y^{0}\right) \\
& \cdot\left(P_{0 \mid 0} \cdot f_{Y}\left(y^{0} \mid D=0, Z=0\right)-P_{0 \mid 1} \cdot \int h_{n}^{0} d y^{1}\right), \\
h_{d f}^{1} & =h_{d f}^{0}=g\left(y^{1}\right) \cdot g\left(y^{0}\right),
\end{aligned}
$$

where the parameters

$$
\begin{aligned}
& \alpha_{a}^{1}=\frac{E\left(Y \mid D=1, Z=1, Y \geq y_{1-q_{1}}\right)-E(Y \mid D=1, Z=0)}{E\left(Y \mid D=1, Z=1, Y \geq y_{1-q_{1}}\right)-E\left(Y \mid D=1, Z=1, Y \leq y_{q_{1}}\right)}, \\
& \alpha_{n}^{0}=\frac{\left.E\left(Y \mid D=0, Z=0, Y \geq y_{1-q_{0}}\right)-E(Y \mid D=0, Z=1)\right)}{E\left(Y \mid D=0, Z=0, Y \geq y_{1-q_{0}}\right)-E\left(Y \mid D=0, Z=0, Y \leq y_{q_{0}}\right)},
\end{aligned}
$$


are set so that the Assumption 2 holds.

Notice that by setting $\operatorname{Pr}(T=t \mid Z)=\operatorname{Pr}(T=t)=\pi_{t}, t=, a, c, n$ as in Table 2, Assumptions 1 , and 3 are immediately satisfied. It is left to show that the functions in (3): (a) are proper probability conditional densities, (b) satisfy Assumption 2 and (c) are compatible with the observed probability distribution $(Y, D, Z)$.

(a) The inequalities in (2) imply that parameters in (4) lie between $[0,1]$. This fact guarantees that $h_{a}^{1}, h_{a}^{0}, h_{n}^{1}$ and $h_{n}^{0}$ are proper densities because their marginals are convex combinations of proper probability densities. We now inspect the non-negativity of the marginal distribution of $h_{c}^{1}$ w.r.t. $y^{1}$ as non-negativity of the marginal distribution of $h_{c}^{1}$ w.r.t. $y^{0}$ follows similarly.

$$
\begin{aligned}
\int h_{c}^{1} d y^{0} & =\left(P_{1 \mid 1}-P_{1 \mid 0}\right)^{-1} \cdot\left(P_{1 \mid 1} \cdot f_{Y}\left(y^{1} \mid D=1, Z=1\right)-P_{1 \mid 0} \cdot \int h_{a}^{1} d y^{0}\right) \\
& =\left(P_{1 \mid 1}-P_{1 \mid 0}\right)^{-1} \cdot f_{Y}\left(y^{1} \mid D=1, Z=1\right) \cdot P_{1 \mid 1} \cdot\left(\left(1-\alpha_{a}^{1}\right) \cdot I\left\{Y \leq y_{q_{1}}\right\}\right. \\
& \left.+\alpha_{a}^{1} \cdot I\left\{Y \geq y_{1-q_{1}}\right\}+1 \cdot I\left\{y_{q_{1}}<Y<y_{1-q_{1}}\right\}+0 \cdot I\left\{y_{1-q_{1}}<Y<y_{q_{1}}\right\}\right) \geq 0 .
\end{aligned}
$$

where $I\{A\}$ is the indicator function of a set $A$. Non-negativity follows from $\alpha_{a}^{1}$ and $\alpha_{n}^{0}$ lying in $[0,1]$.

(b) Note that Assumption 2 is satisfied by construction for the compliers as $h_{c}^{1}=h_{c}^{0}$. We now show that Assumption 2 is satisfied for the always takers, the proof for the never takers is symmetric and thus omitted.

$$
\begin{aligned}
\iint y^{1} h_{a}^{1} d y^{1} d y^{0} & =\iint y^{1}\left(\alpha_{a}^{1} f_{Y}\left(y^{1} \mid D=1, Z=1, Y \leq y_{q_{1}}\right)\right. \\
& \left.+\left(1-\alpha_{a}^{1}\right) f_{Y}\left(y^{1} \mid D=1, Z=1, Y \geq y_{1-q_{1}}\right)\right) d y^{1} d y^{0} \\
& =\alpha_{a}^{1} E\left(Y \mid D=1, Z=1, Y \leq y_{q_{1}}\right)+\left(1-\alpha_{a}^{1}\right) E\left(Y \mid D=1, Z=1, Y \geq y_{1-q_{1}}\right) \\
& =E(Y \mid D=1, Z=0)=\iint y^{1} h_{a}^{0} d y^{1} d y^{0} .
\end{aligned}
$$


(c) Compatibility with the observed probability distribution of $(Y, D, Z)$ follows immediately by the following equalities

$$
\begin{aligned}
& P_{1 \mid 1} \cdot f_{Y}(y \mid Z=1, D=1)=P_{1 \mid 0} \int h_{a}^{1} d y^{0}+\left(P_{1 \mid 1}-P_{1 \mid 0}\right) \int h_{c}^{1} d y^{0}, \\
& P_{1 \mid 0} \cdot f_{Y}(y \mid Z=0, D=1)=P_{1 \mid 0} \int h_{a}^{0} d y^{0}, \\
& P_{0 \mid 1} \cdot f_{Y}(y \mid Z=1, D=0)=P_{0 \mid 1} \int h_{n}^{1} d y^{1}, \\
& P_{0 \mid 0} \cdot f_{Y}(y \mid Z=0, D=0)=P_{0 \mid 1} \int h_{n}^{0} d y^{1}+\left(P_{1 \mid 1}-P_{1 \mid 0}\right) \int h_{c}^{0} d y^{1} .
\end{aligned}
$$

Part (ii)

For any given probability distribution of $(Y, D, Z)$ that satisfy the inequalities in (2), consider the specification (3) for $h_{t}^{z}$, but with parameters $\alpha_{a}^{1}$ and $\alpha_{n}^{0}$ in $[0,1]$, that are different from those in (4). This specification satisfies (5), so that $h_{t}^{z}$ are proper probability distributions while (7) also holds, so that this choice of $h_{t}^{z}$ is compatible with the observed distribution of $(Y, D, Z)$. At the same time, Assumption 2 is violated as the third equation in (6) does not hold.

\section{References}

Angrist, Joshua, Guido Imbens and Donald Rubin, "Identification of Causal Effects using Instrumental Variables," Journal of American Statistical Association 91 (1996), 444-472 (with discussion).

Horowitz, Joel L and Charles F Manski, “Identification and Robustness with Contaminated and Corrupted Data," Econometrica 63 (1995), 281-302.

Huber, Martin, Lukas Laffers and Giovanni Mellace, "Sharp IV bounds on average treatment effects on the treated and other populations under endogeneity and noncompliance," Working Paper (2014).

Huber, Martin and Giovanni Mellace, “Testing instrument validity for LATE identification based on inequality moment constraints," forthcoming in the Review of Economics and Statistics (2013).

Imbens, Guido and Joshua Angrist, "Identification and estimation of local average treatment effects," Econometrica 62 (1994), 467-475.

Kitagawa, Toru, "A test for instrument validity," CeMMAP working paper 34/14 (2014). 